\title{
Eignung und Anwendung von GKV-Routinedaten zur Überprüfung von Versorgungsleitlinien am Beispiel der Indikation Linksherzinsuffizienz
}

\section{Suitability and Usability of Claims Data for Review of Guidelines for the Treatment of Chronic Heart Failure}

Autoren

Institute

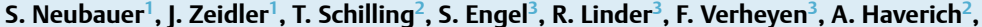
J.-M. G. von der Schulenburg

${ }^{1}$ Center for Health Economics Research Hannover (CHERH), Leibniz Universität Hannover, Hannover

${ }^{2}$ Klinik für Herz-, Thorax-, Transplantations- und Gefäßchirurgie (HTTG), Medizinische Hochschule Hannover, Hannover ${ }^{3}$ WINEG, Techniker Krankenkasse, Hamburg
Schlüsselwörter

- GKV-Routinedaten

- Versorgungsleitlinien

- Herzinsuffizienz

- Leitlinienadhärenz

- Kardiologie

Key words

- claims data

- guidelines

- heart failure

- guideline adherence

- cardiology
Bibliografie

Dol http://dx.doi.org/

10.1055/s-0042-100727

Online-Publikation: 7.4.2016

Gesundheitswesen 2016;

78 (Suppl. 1): e135-e144

(c) Georg Thieme Verlag KG

Stuttgart · New York

ISSN 0949-7013

Korrespondenzadresse Sarah Neubauer, Dipl.-Ök. Center for Health Economics Research Hannover (CHERH) Leibniz Universität Hannover Königsworther Platz 1 30167 Hannover sn@ivbl.uni-hannover.de

\section{Zusammenfassung}

$\nabla$

Hintergrund: Leitlinien spielen eine zentrale Rolle in der Medizin, fraglich ist jedoch, inwiefern diese Empfehlungen im Versorgungsalltag angewendet und unter welchen Voraussetzungen GKV-Routinedaten zur Überprüfung der Leitlinienadhärenz genutzt werden können.

Methodik: Chancen und Limitationen der Routinedaten sowie Voraussetzungen an das Indikationsgebiet und an die Leitlinien selbst wurden bezüglich der Leitlinienüberprüfung anhand eines Expertenpanels analysiert und kritisch gewürdigt. Darauffolgend wurden abbildbare Empfehlungen anhand realer Routinedaten der Techniker Krankenkasse (TK) analysiert, um die Ergebnisse zu untermauern.

Ergebnisse: Die Studienpopulation umfasst 104236 Patienten. Mit Einschränkungen eignen sich GKV-Routinedaten, um Leitlinienadhärenz abzubilden. Es zeigt sich, dass bei den Beta-Rezeptorenblockern die höchste Versorgungsquote mit 70,5\% erreicht wird. Im Gegensatz dazu wurden 56,4\% der Patienten mit reinen ACE-Hemmern sowie ACE-Kombipräparaten versorgt.

Schlussfolgerung: Um Leitlinienkonformität mit Routinedaten abbilden zu können, müssen eine Reihe von Voraussetzungen bezüglich der Datengrundlage, des Indikationsgebietes sowie der Leitlinien selbst erfüllt sein. Leitlinienempfehlungen, die bspw. auf klinischen Daten basieren, können nicht abgebildet werden. Trotz dieser Einschränkungen stellen Routinedaten ein geeignetes Instrument zur Überprüfung ausgewählter Leitlinienempfehlungen dar. Sie zeigen jedoch auch Indizien dafür auf, dass die Versorgungsquoten bei der Pharmakotherapie und weiteren medizinischen sowie diagnostischen Interventionen im Kontext der Leitlinienempfehlungen erhöht werden könnten.

\section{Abstract \\ $\nabla$}

Background: Guidelines have special importance in medicine, however, it is questionable to what extent these recommendations are applied in daily care, and under which conditions claims data can be used for verification of guideline adherence.

Method: Advantages and limitations of claims data for verification of guidelines compliance in the therapeutic area as well as the guidelines themselves were analysed and critically assessed. To substantiate these results, claims data of a major German health insurance fund (Techniker Krankenkasse) were analysed.

Results: 104236 patients were identified. With certain limitations, claims data are useful for verifying guideline adherence; it could be shown that in pharmacotherapy the beta-adrenergic receptor blocker was used to the highest extent (70.5\%). In contrast, only $56.4 \%$ of patients were treated with pure ACE inhibitors and ACE combined preparation.

Conclusion: In order to validate guideline adherence by means of claims data analyses, a number of conditions relating to the database, the therapeutic area and the guidelines themselves have to be considered. Guideline recommendations, which, for example, are based on clinical data, cannot be reviewed by using claims data. Despite these limitations, claims data provide a suitable tool for reviewing selected guideline recommendations. They show that the current use of pharmacotherapy as well as clinical and diagnostic interventions might be increased in accordance with the guideline recommendations. 


\section{Einleitung und Hintergrund}

Die Herzinsuffizienz geht mit einer unzureichenden Funktion des Herzens einher, bei der das Herz nicht mehr imstande ist, eine den Anforderungen entsprechende Förderleistung zu erbringen. Von der Herzinsuffizienz sind in Deutschland zwischen 800000 und 1600000 Personen betroffen [1]. Die Häufigkeit (Prävalenz) und die Neuerkrankungsrate (Inzidenz) der chronischen Herzinsuffizienz sind stark vom Alter des Patienten abhängig [2]. Laut Statistischem Bundesamt stellt die Herzinsuffizienz aktuell die dritthäufigste Todesursache in Deutschland dar [4] und verursachte Kosten in Höhe von 3,2 Mrd. Euro im Jahr 2008 [5], sodass die Herzinsuffizienz auch von ökonomischer Relevanz ist. Infolge des demografischen Wandels und durch verbesserte Überlebenschancen bei den verursachenden Erkrankungen - z. B. nach einem Herzinfarkt - wird diese Zahl in Zukunft voraussichtlich weiter zunehmen und die damit verbundenen Gesundheitsausgaben weiter ansteigen lassen [3]. In den letzten Jahren haben die Routinedaten der gesetzlichen Krankenversicherung (GKV) in der Gesundheitsökonomie und Versorgungsforschung an Bedeutung gewonnen [6]. Die zunehmende wissenschaftliche Nutzung dieser Sekundärdatenquelle ist auf eine Reihe von spezifischen Vorteilen, wie z.B. der Alltagsbezug, die kostengünstige Generierung der Daten, ein meist großes Studienkollektiv und die Einbeziehung von Personengruppen, die sonst üblicherweise eher schwer durch eine Primärdatenerhebung erfasst werden können (Kinder, Schwerstkranke, Demenzerkrankte oder Bewohner von Altenheimen), zurückzuführen. Neben diesen Vorteilen besitzen GKV-Routinedaten jedoch auch spezifische Limitationen [7], da die Daten für einen anderen Verwendungszweck - der Abrechnung - erhoben werden. Die GKV-Routinedaten können somit auch lediglich Leistungen erfassen, die auch über die GKV abgerechnet wurden. Weiterhin sind z.B. Leistungen im ambulanten Bereich nur quartalsweise und Arzneimittel durch die pauschalierte Abrechnung des DRG-Systems gar nicht abbildbar. Diese Limitationen können einen differenzierten Abgleich zwischen Leitlinienempfehlungen und der Versorgungsrealität einschränken.

Leitlinien spielen eine zentrale Rolle in der Medizin, da sie die aktuelle wissenschaftliche sowie medizinische Evidenz widerspiegeln und Handlungsempfehlungen an die behandelnden Ärzte geben. Fraglich ist in diesem Zusammenhang, inwiefern diese Empfehlungen im Versorgungsalltag angewendet werden bzw. angewendet werden können [8].

Bislang gibt es nur wenige Studien, die sich mit der Überprüfung der Anwendung und Anwendbarkeit von Leitlinien in der Versorgungspraxis beschäftigen [9-11]. So untersuchten Swart und Willer anhand von Routinedaten der AOK Baden-Württemberg für Versicherte mit Wohnort Kinzigtal Arthrose-Leitlinien verschiedener Fachgesellschaften. Sie stellten fest, dass, soweit die untersuchten Leitlinien sich anhand von Routinedaten operationalisieren lassen, ihnen weitgehend gefolgt wird. Dennoch sahen sie Herausforderungen in der Nutzung von Routinedaten, da wesentliche Elemente der Leitlinien nicht abgebildet werden konnten, die Beobachtungszeiträume häufig kurz sind oder die Validität der Abrechnungsdaten partiell unklar ist [9]. Laux et al. betrachteten in ihrer Analyse mithilfe des CONTENT-Registers ausschließlich die Qualitätsindikatoren der Nationalen Versorgungsleitlinie (NVL) für chronische Herzinsuffizienz [3,10]. Dieses Morbiditätsregister beinhaltet eine Volldatenerhebung im hausärztlichen Bereich mit einem erweiterten Dokumentationskonzept. Andere Empfehlungen und Leistungssektoren fanden jedoch keine Berücksichtigung. Auch sie sahen Herausforderungen in der Abbildbarkeit der Leitlinienkonformität, da für 5 von den insgesamt 9 Indikatoren notwendige Informationen in den Routinedaten nicht enthalten sind [10]. Die Leitlinienadhärenz im europäischen Vergleich analysierten Flesch et al. Dabei nutzten sie Praxisdaten von niedergelassenen Kardiologen der MAHLERStudie zur Abbildung der Pharmakotherapie und ihren Einfluss auf die Hospitalisierungshäufigkeit und -dauer. Sie zeigten, dass eine leitlinienkonforme Therapie die Krankenhauseinweisungshäufigkeit bei Herzinsuffizienzpatienten senkt. Routinedaten der gesetzlichen Krankenversicherung fanden keine Berücksichtigung. Die Deutsche Angestellten Krankenkasse (DAK) hingegen evaluierte anhand der Indikation Herzinsuffizienz mithilfe von Routinedaten ihres Disease Management Programms (DMP). In der Studie wird auf eine Unterversorgung mit Betablockern hingewiesen. Über die generelle methodische Eignung von GKV-Routinedaten werden jedoch keine Aussagen getroffen. Auch die DAK konzentrierte sich auf die Pharmakotherapie und untersuchte keine weiteren Leitlinienempfehlungen [12].

Bislang wurden GKV-Routinedaten nur selten genutzt, um einen umfassenden Vergleich mit Leitlinien durchzuführen [13]. Die vorliegende Studie stellt eine umfassende Untersuchung der gesamten NVL für chronische Herzinsuffizienz mithilfe von GKV-Routinedaten dar. Die hohe medizinische und gesundheitsökonomische Bedeutung der Herzinsuffizienz erfordern ein unablässiges Bemühen um eine möglichst optimale Diagnostik und Therapie dieser Volkskrankheit. Zudem ist die Herzinsuffizienz neben Diabetes die einzige Erkrankung, bei der mittels ICD-10-Kodierung der Schweregrad kodiert wird. Dies ermöglicht eine differenziertere Überprüfung der Anwendbarkeit von Routinedaten als bei anderen Erkrankungen. Des Weiteren ist für diese Indikation eine ausführliche Versorgungsleitlinie - die NVL - vorhanden. Die NVL ist eine Weiterentwicklung bestehender Literatur wie Hoppe et al. [14] sowie der Leitlinien der Deutschen Gesellschaft für Allgemeinmedizin und Familienmedizin (DEGAM) [1] und wurde im Dezember 2009 zum ersten Mal verabschiedet und regelmäßig aktualisiert. Die Leitlinien und Qualitätsindikatoren bilden eine Basis für die Einschätzung der Versorgungsqualität und die Verbesserung von Versorgungsprozessen [10].

Die vorliegende Studie untersucht daher eine Sequenz von 2 Fragestellungen: Zunächst wird geprüft, unter welchen Voraussetzungen sich GKV-Routinedaten zur Überprüfung von Leitlinien eignen. Es folgt die Ermittlung der Leitlinienadhärenz bei Diagnostik und Therapie von Herzinsuffizienzpatienten im Versorgungsalltag.

\section{Methodik \\ $\nabla$}

\section{Datengrundlage}

Um die Nutzbarkeit von GKV-Routinedaten zur Überprüfung von ärztlichen Leitlinien zu bewerten, wurden Abrechnungsdaten der Techniker Krankenkasse (TK) als eine der größten Krankenkassen Deutschlands verwendet [15]. Zunächst wurden alle Versicherten identifiziert, bei denen von 2008 bis 2011 mindestens einmal die Diagnose Herzinsuffizienz (ICD-10: I50,1-I50,9) dokumentiert wurde. Als Herzinsuffizienz-Patienten wurden Versicherte definiert, die im Studienzeitraum mindestens eine stationäre Haupt- oder Nebenentlassungsdiagnose und/oder eine gesicherte ambulante Diagnose I50,1-I50,9 hatten. Neben den durchgängig Versicherten wurden ebenfalls Herzinsuffizi- 
enz-Patienten berücksichtigt, die in dem Studienzeitraum verstorben sind. Um einen Nachbeobachtungszeitraum von mindestens 2 Quartalen (einem halben Jahr) zu gewährleisten, wurden Individuen, die ihr Indexereignis nach dem dritten Quartal 2011 hatten, von der Analyse ausgeschlossen. Gleiches gilt für die Verstorbenen, deren Tod innerhalb des jeweils definierten Nachbeobachtungszeitraums lag, da durch den Tod dieser Analysezeitraum zensiert ist.

Obwohl sich die Leitlinienempfehlungen zur sektorenübergreifenden Versorgung auf Patienten mit Links- und Globalherzinsuffizienz inklusive akuter Dekompensationen beziehen, wurde die Untersuchung auf Patienten mit Linksherzinsuffizienz beschränkt, da die „globale Herzinsuffizienz“ unter die „sekundäre Rechtsherzinsuffizienz“ in der ICD-10-Kodierung fällt und somit nicht eindeutig abgrenzbar ist. Die ICD-10-Codes I50,00 „primäre Rechtsherzinsuffizienz“, I50,01 „sekundäre Rechtsherzinsuffizienz" und I50,9 "nicht näher bezeichnete/definierte Herzinsuffizienz" wurden von der detaillierteren Analyse ausgeschlossen.

Die Linksherzinsuffizienz kann gemäß der Unterdiagnosen I50,11-I50,14 in Anlehnung an die New York Heart Association (NYHA) Klassifikationen I-IV in unterschiedliche Beschwerdegrade differenziert werden. Patienten mit einer nicht näher definierten Linksherzinsuffizienz (ICD-10: I50,19) wurden ausgeschlossen, da für die Analysen der Schweregrad eine entscheidende Rolle spielt. Zusätzlich wurden Versicherte ausgeschlossen, die im ambulanten Bereich lediglich eine vereinzelte Verdachts- oder „Zustand nach“-Diagnose hatten, um sicherzustellen, dass die identifizierten Patienten auch gesicherte Herzinsuffizienzpatienten sind.

In anonymisierter Form wurden sowohl ambulante Abrechnungsdaten, Daten der stationären Versorgung, Angaben zur Rehabilitation und zur Arbeitsunfähigkeit als auch Arznei-, Heil- und Hilfsmitteldaten sowie Stammdaten der Versicherten analysiert.

\section{Studiendesign}

Um notwendige Voraussetzungen von GKV-Routinedaten zur Überprüfung der Leitlinienkonformität zu ermitteln, wird zunächst differenziert darauf eingegangen, welche Eigenschaften bezüglich der Datengrundlage, bei den Indikationsgebieten und in den Leitlinien erfüllt sein müssen. Dabei gibt es Empfehlungen, die gut und umfassend unter gewissen Annahmen oder Limitationen, sowie nicht über GKV-Routinedaten abbildbar sind.

\section{Die Nationale Versorgungsleitlinie}

Die NVL gliedert sich in 16 Themenbereiche, die 127 Empfehlungen zu Prävention, Diagnostik und Therapie sowie Komorbiditäten, geriatrische Besonderheiten oder psychosoziale Aspekte enthalten [3].

In dieser Studie wurden alle Empfehlungen der NVL in einem Expertenkonsens systematisch auf ihre jeweilige Abbildbarkeit in den Routinedaten untersucht. Darauffolgend wurde die Anwendung der jeweiligen Empfehlungen in der Versorgungsrealität anhand der Qualitätsindikatoren (EKG, Echokardiografie, 2 Indikatoren bzgl. allgemeiner Behandlungsstrategie, ACE-Hemmer, Beta-Rezeptorenblocker, Versorgungsmanagement und Nahtstellen), Pharmakotherapie insgesamt, Komorbiditäten und weiteren Therapieelementen wie Impfschutz, Verordnung von Rehabilitation bei stabiler Herzinsuffizienz (NYHA I-III), Kardiale Resynchronisationstherapie (CRT) und Einsetzen eines implantierbaren Cardioverter-Defibrillators nach überlebten Herzrhythmusstörungen überprüft. Mittels Ziffern des Einheitlichen
Bewertungsmaßstabs (EBM) sowie der Operationen- und Prozedurenschlüsseln (OPS) können die medizinischen Maßnahmen abgebildet und analysiert werden.

\section{Indexereignis}

Für die Standardisierung des Beobachtungszeitraums wurde für jeden Versicherten ein sogenanntes Indexereignis erstellt. Dies ist definiert als das Quartal und Jahr des ersten Auftretens einer ambulanten oder stationären Herzinsuffizienz-Diagnose innerhalb des Beobachtungszeitraums. Dieses Indexereignis ist jedoch nicht als Inzidenz-Kriterium im gesamten Krankheitsverlauf zu interpretieren, sondern bezieht sich nur auf den Beobachtungszeitraum.

Ausgehend von dem individuellen Indexereignis wurden Zeiträume für die Analyse der Leitlinienkonformität gewählt ( $\bullet$ Tab. 1). Der kürzere Beobachtungszeitraum für das EKG und die Echokardiografie wurde festgelegt, da diese Leistungen zur Erstellung und Verifizierung der Herzinsuffizienz-Diagnose genutzt wurden.

Einen Überblick über den Nachbeobachtungszeitraum und die Abbildbarkeit der Empfehlungen gibt $\bullet$ Tab. 1.

\section{Arzneimittelverordnungen}

Anhand der Arzneimitteldaten können medikamentöse Behandlungsstrategien, stratifiziert nach NYHA-Schweregraden, abgebildet werden. ACE-Hemmer sind laut der NVL bei jedem Schweregrad der Herzinsuffizienz empfohlen, Beta-Rezeptorenblocker sollen hingegen beim Schweregrad I nur nach Auftreten eines Myokardinfarkts oder bei Vorliegen einer Hypertonie verschrieben werden. Außerdem wird der Einsatz von Beta-Rezeptorenblockern bei Patienten mit Asthma nicht empfohlen [3]. Zur NYHA-Einstufung wurde der höchste dokumentierte Schweregrad, d.h. der maximale durch eine ICD-10-Diagnose abgebildete NYHA-Status, pro Quartal genutzt. In dieser Analyse wurden Ein- und Ausschlussmedikationen sowie Kontraindikationen untersucht. Das Verordnungsdatum stellt für die Analyse die am besten geeignete Variable aus den GKV-Arzneimitteldaten dar, da zwischen Verschreibungs- und Abgabedatum 30 Tage, ggf. auch noch längere Zeiträume, liegen können.

Die Komorbiditäten Hypertonie, Angina Pectoris, Niereninsuffizienz, Diabetes mellitus, Asthma und COPD sind explizit in der NVL erwähnt und werden nach Häufigkeit ihres Auftretens analysiert. Des Weiteren stellen sie bei einigen Medikationsstrategien der Herzinsuffizienz eine Kontraindikation dar. Falls eine Kontraindikation vorlag, wurde geprüft, ob diesen Patienten trotzdem nicht empfohlene Arzneimittel verordnet wurden.

Für die Pharmakotherapie-Analyse gingen nur die genannten Kontraindikationen in die Untersuchung mit ein, die vor dem Indexquartal oder innerhalb der 3 Folgequartale aufgetreten sind. Kontraindikationen, die nach dem Analysezeitraum von einem Jahr nach dem Indexereignis diagnostiziert wurden, wurden nicht berücksichtigt.

\section{Regelmäßige Arztkontakte}

In den Leitlinien werden „regelmäßige Arztkontakte“ empfohlen. Eine Konsultation eines Facharztes wird in der Leitlinie weder direkt gefordert, noch wird eine zeitliche Komponente genannt, dennoch lassen sich spezifische Unterschiede der hausärztlichen und fachärztlichen Versorgung untersuchen. Dazu wurde die 9-stellige lebenslange Arztnummer (LANR) der Kassenärztlichen Vereinigung (KV), in anonymisierter Form, herangezogen, wobei die achte und neunte Ziffer die Facharztrichtung angibt. Die ersten 7 Stellen gelten „lebenslang“ 
Tab. 1 Überblick über den Nachbeobachtungsraum und die Abbildbarkeit der jeweiligen zu untersuchenden Indikatoren und Empfehlungen.

\begin{tabular}{|c|c|c|}
\hline Qualitätsindikatoren und Empfehlungen: & Analysezeitraum: & Abbildbarkeit durch: \\
\hline \multicolumn{3}{|l|}{ Diagnostik } \\
\hline EKG & Indexquartal + Folgequartal & EBM: 03320, 04320, 27320, 27321 \\
\hline Echokardiografie & Indexquartal + Folgequartal & $\begin{array}{l}\text { EBM, ambulant: 13545, 13550, 33020, 33021, 33022, 33030, } 33031 \\
\text { OPS, stationär: 3-031, 3-052 }\end{array}$ \\
\hline \multicolumn{3}{|l|}{ Pharmakotherapie } \\
\hline ACE-Hemmer & Indexquartal + 3 Folgequartale & ATC-Code: C09A- \\
\hline Beta-Rezeptorenblocker & Indexquartal + 3 Folgequartale & ATC-Code: $\mathrm{CO7}$ \\
\hline Pharmakotherapie allgemein \& Kontraindikation & Indexquartal + 3 Folgequartale & ATC-Code/ ICD-10 \\
\hline \multicolumn{3}{|l|}{ Komorbiditäten und Kontraindikationen } \\
\hline Hypertonie & \multirow{6}{*}{$\begin{array}{l}\text { Zeitraum vor Indexquartal oder } \\
\text { innerhalb der } 3 \text { Folgequartale }\end{array}$} & ICD-10: 110 \\
\hline Angina Pectoris & & ICD-10: 120.0 \\
\hline Niereninsuffizienz & & ICD-10: N18, N19 \\
\hline Diabetes mellitus & & ICD-10: E10, E11, E12, E13, E14 \\
\hline Asthma & & ICD-10: J45, J46 \\
\hline COPD & & ICD-10: J44 \\
\hline \multicolumn{3}{|l|}{ weitere Therapieelemente: } \\
\hline Grippeschutzimpfung & Indexquartal + 3 Folgequartale & EBM-Ziffern und Sonderziffern der KVen \\
\hline $\begin{array}{l}\text { Verordnung von Rehabilitation bei stabiler } \\
\text { Herzinsuffizienz (NYHA I-III) }\end{array}$ & $\begin{array}{l}\text { Gesamter Zeitraum nach dem } \\
\text { Indexereignis }\end{array}$ & EBM-Ziffer: 01611 \\
\hline $\begin{array}{l}\text { Einsetzen eines implantierbaren Cardiover- } \\
\text { ter-Defibrillators nach überlebten Herzrhyth- } \\
\text { musstörungen }\end{array}$ & $\begin{array}{l}\text { Gesamter Zeitraum nach dem } \\
\text { Herztod }\end{array}$ & OPS-Codes: $5-377.5$ bis. 7 sowie $5-377 . f$ \\
\hline
\end{tabular}

für die gesamte vertragsärztliche Tätigkeit. Sie sind KV-übergreifend, unabhängig vom Status, der Zugehörigkeit zu Berufsausübungsgemeinschaften und dem Tätigkeitsort. Über diese Facharztnummer wurden die Versicherten in die Gruppen „ausschließlich vom Facharzt behandelt“, „ausschließlich vom Hausarzt behandelt“, „Von beiden Arztgruppen behandelt“, und „weder vom Hausarzt noch von einem ausgewählten Facharzt behandelt" unterteilt.

\section{Rehabilitationsmaßnahmen}

Bei einem sich nicht verändernden NYHA-Status ist laut Leitlinie eine Rehabilitationsmaßnahme zu empfehlen. Hierzu wurde analysiert, wie häufig die EBM-Ziffer 01611 („Verordnung von medizinischer Rehabilitation“) abgerechnet wurde. Die Stabilität der Patienten wird in der Leitliniendefinition nicht näher definiert, daher wurde die gesamte Studienpopulation analysiert.

\section{Grippeschutzimpfung}

In der Leitlinie wird empfohlen, dass jeder Patient einmal pro Jahr eine Grippeschutzimpfung erhalten soll. Anhand von spezifischen EBM-Ziffern und Sonderziffern der KVen könnte diese Vorsorgemaßnahme grundsätzlich identifiziert und quantifiziert werden.

\section{Kardiale Resynchronisationstherapie und ICD- Implantation}

Als apparative Therapie wird in der Leitlinie bei NYHA-Status III-IV eine kardiale Resynchronisationstherapie (CRT) angeraten. Die Anzahl herzinsuffizienzbedingter CRT ist nicht explizit aus den Routinedaten ablesbar, da CRT keine eigene spezifische EBM und/oder OPS-Kodierung besitzt. In dieser Untersuchung wird daher nur die Implantation von Defibrillatoren und Schrittmachern erfasst.

Nach Überleben eines plötzlichen Herztodes empfiehlt die Leitlinie das Einsetzen eines implantierbaren Cardioverter-Defibrillators. Daher wurden zunächst alle Patienten mit einem plötzli- chen Herztod anhand der ambulanten und stationären Diagnosedaten identifiziert. Anschließend wurde anhand von OPSCodes überprüft, ob bei diesen Patienten ein Cardioverter-Defibrillator implantiert wurde.

\section{Einfluss von Patientencharakteristika auf die Leitlinienadhärenz}

Um den Einfluss von Alter, Geschlecht, NYHA-Status und Komorbiditäten auf die Leitlinienadhärenz hinsichtlich der medikamentösen Therapie zu prüfen, wurden $\mathrm{Chi}^{2}$, Mann/Whitney U Tests und logistische Regressionsanalysen durchgeführt.

\section{Software}

Die Datenextraktion sowie das Datenmanagement wurden mit der Software SAS Enterprise Guide (Version 4.3) realisiert. Zusätzlich wurden die Softwareprogramme IBM SPSS Statistics Version 20 sowie STATA Version 8 für die deskriptiven statistischen Analysen verwendet.

\section{Ergebnisse}

Die Studienpopulation umfasst 104236 Versicherte und teilt sich, um unterschiedliche Nachbeobachtungszeiträume gewährleisten zu können, in 2 Subgruppen auf ( $\bullet$ Abb. 1). Die erste Subgruppe beinhaltet alle Versicherten, für die ein Nachbeobachtungszeitraum von mindestens 2 Quartalen gewährleistet werden kann (Indexquartal+Folgequartal, $n=98099$ ). Die zweite Subpopulation schließt alle Versicherten ein, die sich zusätzlich zu ihrem Indexquartal für weitere 3 Folgequartale beobachten lassen $(n=85465)$. Das Durchschnittsalter lag für die gesamte Studienpopulation bei 71,3 Jahren und der Anteil an Männern betrug 66\%. 24206 Versicherte sind im Beobachtungszeitraum verstorben. Das durchschnittliche Sterbealter lag bei 77,4 Jahren. 


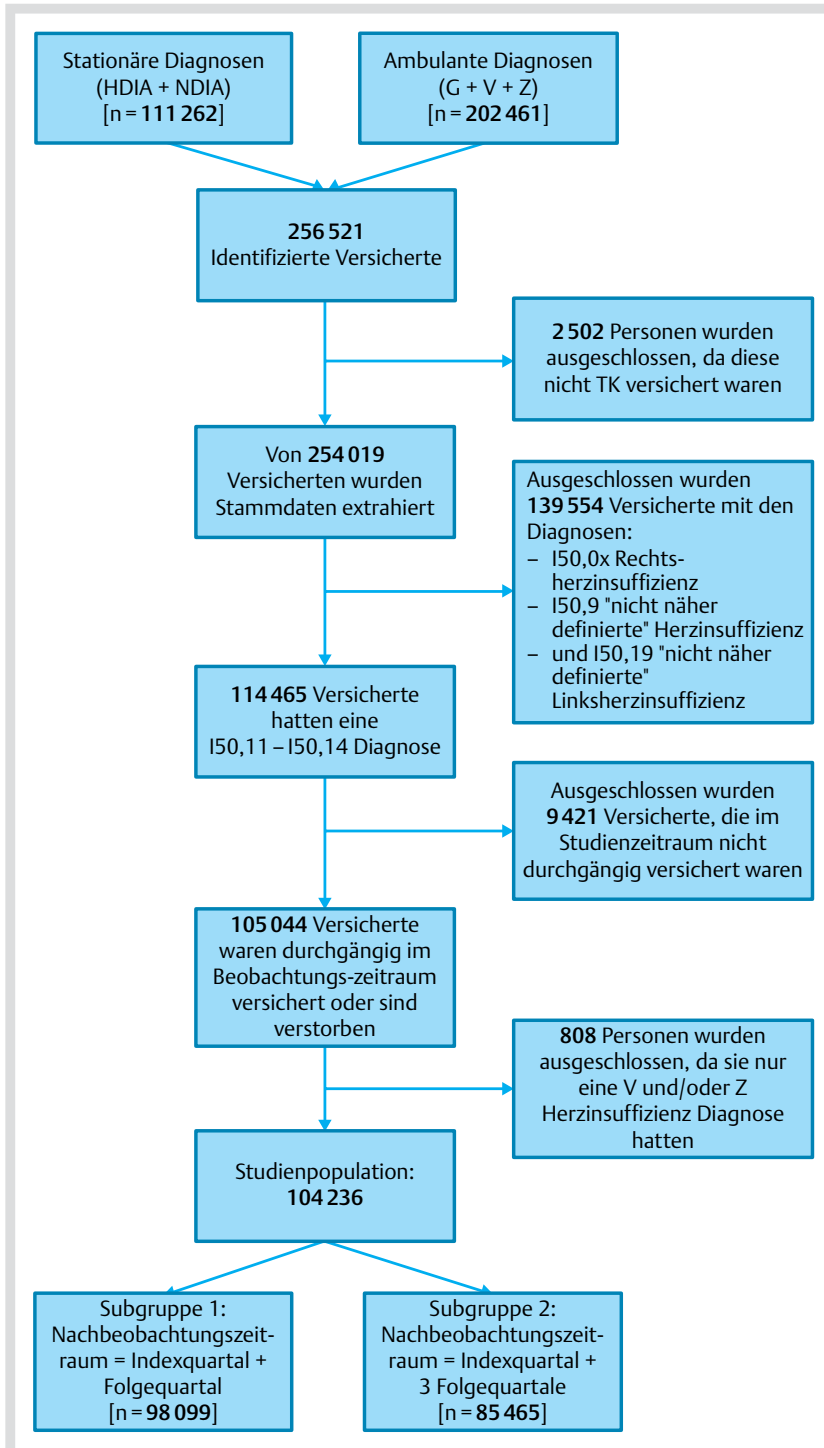

Abb. 1 Identifizierung der Studienpopulation. HDIA = Hauptdiagnose, NDIA= Nebendiagnose, $G$ = gesicherte Diagnose, $\mathrm{V}=$ Verdachtsdiagnose, $\mathrm{Z}=$ Zustand nach Diagnose

\section{Voraussetzungen}

Die Voraussetzungen, um mithilfe von GKV-Routinedaten die Versorgungsleitlinien überprüfen zu können, werden in $\odot$ Tab. 2 und $\odot$ Tab. 3 zusammenfassend und systematisch aufgeführt. 4 von den insgesamt 9 Qualitätsindikatoren der NVL lassen sich auf Basis von GKV-Routinedaten mit unterschiedlicher Schärfe abbilden: EKG-Untersuchungen, Echokardiografiediagnostik, ACE-Hemmer-Verordnung sowie Beta-Rezeptorenblocker-Verordnung. Die restlichen 5 (Dokumentation über Beratung zu körperlicher Aktivität, dokumentierte Beratung zur Gewichtsmessung und Information über die Notwendigkeit, bei kurzfristigem Gewichtsanstieg den Arzt zu informieren; Kardiale Resynchronisation; Verlaufskontrolle sowie Versorgungsmanagement und Nahtstellen) konnten anhand der Datengrundlage sowie der unspezifischen Formulierung der Leitlinie nicht dargestellt werden. Gleiches gilt für die Empfehlungen zu Prävention, klinischen Befunden, Arzt-Patient-Kommunikation, Lebensgewohnheiten sowie persönliche Informationen der Versicherten.

\section{Abgleich der Leitlinienempfehlungen der NVL mit den GKV-Abrechnungsdaten \\ Echokardiografie}

Im Krankenhaus wurden für 14034 (14,3\% der Subgruppe 1) Versicherte im Index- und/oder im Folgequartal mindestens eine Echokardiografie abgerechnet. 40324 (41,1\% der Subgruppe 1) Versicherte wurden im Index- und/oder im Folgequartal mindestens einmal per Echokardiografie im ambulanten Bereich untersucht.

\section{Elektrokardiogramm (EKG)}

Die Abbildung und Analyse der basisdiagnostischen Leistung EKG mittels EBM-Ziffern mit den GKV-Routinedaten ist möglich, sofern diese Leistung nicht pauschal abgerechnet wird. Für 177 Versicherte (0,2\% der Subgruppe 1) wurde ein EKG abgerechnet.

\section{Pharmakotherapie}

Da ein Beobachtungszeitraum von einem Jahr definiert wurde, betrug die Subgruppe für diese Analyse 85465 Individuen (Subgruppe 2), wovon 11921 Versicherte in NYHA-Klasse I, 36201 Patienten in NYHA-Klasse II entfielen und 37343 Personen an einer schweren Herzinsuffizienz (NYHA III-IV) litten.

Tab. 2 Generelle Voraussetzungen für die Routinedaten, das Indikationsgebiet und die Leitlinien.

\begin{tabular}{|c|c|c|c|}
\hline & GKV-Routinedaten & Indikationsgebiet & Leitlinie \\
\hline Datengrundlage & - & $\begin{array}{l}\text { Indikation muss mittels } \\
\text { ICD-10-Kodierung abbildbar sein. }\end{array}$ & $\begin{array}{l}\text { Existenz einer oder mehrerer umfassender Versor- } \\
\text { gungsleitlinien. }\end{array}$ \\
\hline $\begin{array}{l}\text { Handlungsempfeh- } \\
\text { lungen (allgemein) }\end{array}$ & $\begin{array}{l}\text { Die Empfehlungen müssen mittels } \\
\text { EBM-Ziffern, ATC- und OPS- Codes } \\
\text { sowie ICD-10-Kodierung abbildbar } \\
\text { sein. }\end{array}$ & $\begin{array}{l}\text { Handlungsempfehlungen müssen } \\
\text { speziell für das Indikationsgebiet } \\
\text { beschrieben sein. }\end{array}$ & $\begin{array}{l}\text { Handlungsempfehlungen dürfen nicht auf klinischen } \\
\text { Parametern basieren, da diese mittels Routinedaten } \\
\text { nicht abbildbar sind. }\end{array}$ \\
\hline Stichprobengröße & $\begin{array}{l}\text { Krankenkasse muss eine gewisse } \\
\text { Größe haben, um eine ausreichend } \\
\text { große Datenmenge für die Studien- } \\
\text { population zu besitzen. }\end{array}$ & $\begin{array}{l}\text { Eine hohe Inzidenz der Erkran- } \\
\text { kung ergibt eine ausreichend } \\
\text { große Stichprobe, um die breite } \\
\text { Versorgungspraxis mithilfe von } \\
\text { GKV-Routinedaten zu analysieren. }\end{array}$ & - \\
\hline Einheiten & $\begin{array}{l}\text { Konkret formulierte Empfehlungen } \\
\text { wie z. B. „jährlich“ sind gut mithilfe } \\
\text { der Routinedaten abbildbar. }\end{array}$ & - & $\begin{array}{l}\text { Zeitliche und mengenmäßige Angaben müssen } \\
\text { konkret formuliert sein. Unpräzise Ausdrücke wie } \\
\text { „regelmäßig“ oder „stabile Patienten“ sind in der } \\
\text { NVL nicht ausreichend definiert. }\end{array}$ \\
\hline
\end{tabular}


Tab. 3 Übersicht der Voraussetzungen zur Überprüfung von leitliniengerechter Versorgung für die Routinedaten, dem Indikationsgebiet und der Leitlinien.

\begin{tabular}{|c|c|c|c|c|}
\hline Aspekt & $*$ & GKV-Routinedaten & Indikationsgebiet & Leitlinie \\
\hline $\begin{array}{l}\text { Schweregrad } \\
\text { der Krankheit }\end{array}$ & $\sim$ & - & $\begin{array}{l}\text { Schweregrad muss } \\
\text { als Unterkategorie } \\
\text { oder mittels eigener } \\
\text { ICD-10-Kodierung } \\
\text { abbildbar sein. }\end{array}$ & $\begin{array}{l}\text { Unterschiedliche Behandlungsmaß- } \\
\text { nahmen müssen für unterschied- } \\
\text { liche Schweregrade vorliegen und } \\
\text { beschrieben sein }\end{array}$ \\
\hline $\begin{array}{l}\text { Haus- und } \\
\text { Facharzt }\end{array}$ & + & $\begin{array}{l}\text { Lebenslange Arztnummer (LANR) kann bei der Identifizie- } \\
\text { rung zwischen HA und FA herangezogen werden. } \\
\text { Die Anzahl der Arztbesuche sowie die Reihenfolge } \\
\text { innerhalb eines Quartals sind lediglich unter bestimmten } \\
\text { Annahmen möglich (Verknüpfung amb. Diagnose- sowie } \\
\text { Leistungsdaten). }\end{array}$ & - & $\begin{array}{l}\text { Die Leitlinie muss klare Zuständigkeiten } \\
\text { von Fach-und Hausarzt formulieren. }\end{array}$ \\
\hline $\begin{array}{l}\text { Pharmako- } \\
\text { therapie }\end{array}$ & + & $\begin{array}{l}\text { Pharmakotherapie lässt sich mithilfe der GKV-Routineda- } \\
\text { ten sehr gut abbilden, da die ATC-Codes, DDD usw. in den } \\
\text { GKV-Routinedaten vorliegen. } \\
\text { Durch die ambulanten und stationären Diagnosedaten } \\
\text { können auch Kontraindikationen durch die ICD-10- } \\
\text { Kodierung aufgedeckt \& abgebildet werden. } \\
\text { Annahmen müssen getroffen werden, wenn es um } \\
\text { Dosierungsempfehlungen pro Körpergewicht geht. }\end{array}$ & $\begin{array}{l}\text { Unterschiedliche } \\
\text { Medikationsmus- } \\
\text { ter abhängig vom } \\
\text { Schwergrad formu- } \\
\text { liert. }\end{array}$ & $\begin{array}{l}\text { Entsprechende anatomisch-therapeu- } \\
\text { tisch-chemische Klassifikationssysteme } \\
\text { (ATC). }\end{array}$ \\
\hline Impfschutz & $\sim$ & $\begin{array}{l}\text { Impfschutz ist nur teilweise abbildbar, da es KV- } \\
\text { spezifische Sonderziffern für Impfungen geben kann. }\end{array}$ & - & $\begin{array}{l}\text { Die Impfintervalle müssen in der Leit- } \\
\text { linie angegeben werden und nicht zu } \\
\text { weit auseinander liegen. }\end{array}$ \\
\hline Beratung & - & $\begin{array}{l}\text { Die Kommunikation zwischen Arzt und Patient sowie die } \\
\text { Beratung ist nicht abbildbar, da diese keine spezifischen } \\
\text { Abrechnungsziffern besitzen. }\end{array}$ & - & $\begin{array}{l}\text { Die regelmäßigen Beratungen sollen un- } \\
\text { terstützend wirken, um die Arzt-Patien- } \\
\text { ten-Beziehung zu verbessern, da häufig } \\
\text { auch Patienten-Leitlinien existieren. }\end{array}$ \\
\hline $\begin{array}{l}\text { Patient und sei- } \\
\text { ne Compliance }\end{array}$ & $\sim$ & $\begin{array}{l}\text { Die Mitarbeit des Patienten z. B. durch die Modifikation } \\
\text { des Lebensstils, die Ausübung von Training und körper- } \\
\text { licher Aktivität ist in den Routinedaten nicht abbildbar. } \\
\text { Gleiches gilt für die Ernährung und das Gewicht. Durch } \\
\text { die DMP-Dokumentation sind gewisse Merkmale erfasst, } \\
\text { dennoch meist noch schlecht dokumentiert. }\end{array}$ & - & $\begin{array}{l}\text { Sollte die Leitlinie eine Umstellung } \\
\text { des Lebensstils empfehlen, ist dies zu } \\
\text { konkretisieren. Dennoch sind Empfeh- } \\
\text { lungen, z. B. die Ernährungsumstellung } \\
\text { oder körperliches Training, nicht in den } \\
\text { GKV-Routinedaten darstellbar. } \\
\text { Die Empfehlungen der Leitlinie sollten, } \\
\text { wenn möglich klar nach unterschiedli- } \\
\text { chen Patientengruppen (Schweregrad } \\
\text { der Krankheit, Kinder/Erwachsene usw.) } \\
\text { unterteilt sein. }\end{array}$ \\
\hline $\begin{array}{l}\text { Krankheitsge- } \\
\text { schichte und } \\
\text { Familie }\end{array}$ & - & $\begin{array}{l}\text { In den Routinedaten können Familienversicherte, z. B. Kin- } \\
\text { der oder Ehepartner dem Mitglied zu geschlüsselt werden, } \\
\text { wenn beide bei derselben Krankenkasse versichert sind. } \\
\text { Andere Familienbeziehungen außerhalb der Familienver- } \\
\text { sicherung sind aber nicht abbildbar, dies führt zu wenigen } \\
\text { Informationen bzgl. der familiären Disposition. } \\
\text { Vorerkrankungen sind nur teilweise abbildbar. Wenn } \\
\text { man in den GKV-Routinedaten lang genug zeitlich zurück } \\
\text { gehen und ein Indexereignis identifizieren kann, könnte } \\
\text { man pre Indexereignis schauen, welche anderen Krankhei- } \\
\text { ten es davor gab. }\end{array}$ & - & - \\
\hline Basisdiagnostik & - & $\begin{array}{l}\text { Ergebnisse von Laborbefunden, Blutbild sowie Urinstatus } \\
\text { sind nicht abbildbar. }\end{array}$ & - & $\begin{array}{l}\text { Die Empfehlungen müssen mittels } \\
\text { EBM-Ziffern sowie OPS Codes abbildbar } \\
\text { sein. }\end{array}$ \\
\hline Prävention & - & $\begin{array}{l}\text { Präventionsangebote sind nicht abbildbar, da es im } \\
\text { besten Fall durch eine Präventionsmaßnahme nicht zu } \\
\text { der ausgewählten Erkrankung kommt und somit keine } \\
\text { ICD-10-Diagnose für den Aufgriff und Identifikation zur } \\
\text { Studienpopulation erhält. }\end{array}$ & - & - \\
\hline $\begin{array}{l}\text { Kontraindika- } \\
\text { tionen }\end{array}$ & + & $\begin{array}{l}\text { Kontraindikationen, die Kontradiagnosen betreffen, } \\
\text { können mittels ICD-10 gut abgebildet werden. Serumkre- } \\
\text { atin-Werte und andere klinische Parameter können mit } \\
\text { GKV-Routinedaten hingegen nicht abgebildet werden. }\end{array}$ & - & $\begin{array}{l}\text { Leitlinien müssen konkrete Kontrain- } \\
\text { dikationen in der Pharmakotherapie } \\
\text { erwähnen. }\end{array}$ \\
\hline
\end{tabular}

Bewertung der Anwendung von GKV-Routinedaten zur Überprüfung von Versorgungsleitlinien: + gute Abbildbarkeit; bedingte bzw. eingeschränkte Abbildbarkeit; - schlechte Abbildbarkeit 


\begin{tabular}{|c|c|c|c|c|c|}
\hline $\mathrm{Cr}_{\text {Arzneimittel }}^{\text {NYHA-Klassen }}$ & NYHA I & NYHA II & \multicolumn{2}{|r|}{ NYHA III } & NYHA IV \\
\hline ACE-Hemmer (C09A- \& C09B-) & \multicolumn{5}{|c|}{$\begin{array}{c}\text { Reine ACE-Hemmer: } 41838(49,0 \%) \\
\text { Kombipräparate: } 12761 \text { (14,9\%) } \\
\text { Reine ACE-Hemmer und/oder Kombipräparate: } 48183 \text { (56,4\%) }\end{array}$} \\
\hline Beta-Rezeptorenblocker (C07-) & $\begin{array}{c}8362(70,1 \%) \\
\text { [davon } 7768(92,9 \%) \\
\text { Versicherte mit } \\
\text { Myokardinfarkt und/oder } \\
\text { Hypertonie] }\end{array}$ & \multicolumn{4}{|c|}{$51853(70,5 \%)$} \\
\hline $\begin{array}{l}\text { Schleifendiuretika } \\
\text { (C03CA01, C03CA04) }\end{array}$ & $\begin{array}{l}4347 \text { ( } 36,5 \% \text { ) Versicherte } \\
\text { bekommen } \\
\text { Schleifendiuretika obwohl } \\
\text { das nicht der Empfehlung } \\
\text { entspricht }\end{array}$ & $16396(45,3 \%)^{*}$ & \multicolumn{3}{|c|}{$22476(60,2 \%)$} \\
\hline $\begin{array}{l}\text { Thiazide } \\
\text { (C03AA03, C03BA10) }\end{array}$ & $\begin{array}{c}1861(15,6 \%) \\
\text { [davon hatten } 1755(94,3 \%) \\
\text { Versicherte Hypertonie] }\end{array}$ & $6029(16,7 \%)^{*}$ & \multicolumn{3}{|c|}{$6841(18,3 \%)$} \\
\hline $\begin{array}{l}\text { Aldosteron-Antagonisten } \\
\text { (C03DA01, C03DA04) }\end{array}$ & $\begin{array}{l}1437(12,1 \%) \text { obwohl das } \\
\text { nicht der Empfehlung } \\
\text { entspricht }\end{array}$ & $\begin{array}{c}5818(16,1 \%) \\
{[963(16,6 \%) \text { mit Myokardinfarkt }]}\end{array}$ & \multicolumn{3}{|c|}{$8233(22,0 \%)$} \\
\hline $\begin{array}{l}\text { AT1-Rezeptorenblocker } \\
\text { (C09CA01, C09CA03, C09CA06) }\end{array}$ & \multicolumn{5}{|c|}{$10264(12,0 \%)^{*}$} \\
\hline \multirow{3}{*}{$\begin{array}{l}\text { Anzahl Versicherte in der } \\
\text { jeweiligen NYHA Klasse }\end{array}$} & \multicolumn{5}{|c|}{85465} \\
\hline & 11921 & \multicolumn{4}{|c|}{73544} \\
\hline & 11921 & 36201 & \multicolumn{3}{|c|}{37343} \\
\hline
\end{tabular}

Abb. 2 Analyse der medikamentösen Stufentherapie nach NYHA-Klassen.

Beta-Rezeptorenblocker waren mit einer Versorgungsquote von 70,5\% über die NYHA-Stadien II-IV hinweg der am häufigsten verschriebene Wirkstoff ( $\bullet$ Abb. 2). Im Gegensatz dazu wurden $56,4 \%$ der Patienten mit reinen ACE-Hemmern sowie ACE-Kombipräparaten versorgt. Die dunkel blauen Flächen kennzeichnen Wirkstoffgruppen-NYHA-Kombinationen, die laut Leitlinie ausgeschlossen werden. Diese Versorgungsquoten liegen mit 36,5\% für die Schleifendiuretika und mit 12,1\% für die Aldosteron-Antagonisten relativ hoch. Die weiß hinlegten Flächen zeigen Wirkstoffgruppen an, die laut NVL in den niedrigeren NYHA-Stadien nur unter bestimmten Voraussetzungen gegeben werden dürfen. Von den 11,921 Versicherten der NYHA Klasse I bekamen 8,362 (70,1\%) Beta-Rezeptorenblocker, obwohl Patienten mit diesem NYHA-Status laut Leitlinie keine Beta-Rezeptorenblocker erhalten sollen. 7768 (92,9\% von den 8,362) Personen hatten jedoch vor ihrem Indexereignis oder 3 Quartale danach einen Myokardinfarkt und/oder litten an Hypertonie, sodass diese Indikationen die Verschreibung dieser Wirkstoffe legitimiert und als leitliniengerecht therapiert eingestuft werden können. Da sich eine Flüssigkeitsretention nicht in einer ICD-10-Diagnose abbilden lässt, wurden in ( $\bullet$ Abb. 2) lediglich die Verordnungen von Diuretika in NYHA-Klasse II abgetragen.

\section{Komorbiditäten}

Eine Hypertonie-Diagnose entfiel auf 93296 Personen (89,5\% der Studienpopulation), 22,3\% der Versicherten wiesen eine Angina Pectoris-Diagnose auf. An Asthma litten 11260 (10,8\%) und an Diabetes mellitus 43950 (42,16\%) der Versicherten. Eine COPD-Diagnose erhielten 26250 Patienten, was einem Anteil von $25,18 \%$ der Versicherten entspricht.
Weitere Therapieelemente

Impfschutz

Bei 38922 Versicherten, d.h. fast 50\% der analysierten Patienten der Subgruppe 2 wurden im Analysezeitraum die spezifischen EBM-Ziffern für eine Grippeschutzimpfung abgerechnet.

\section{Verordnung von Rehabilitation bei stabiler}

Herzinsuffizienz (NYHA I-III)

Insgesamt wurde für 1969 Versicherte (1,9\% der Studienpopulation) eine medizinische Rehabilitation in dem individuellen Beobachtungszeitraum nach dem Indexereignis verordnet. In dem Jahr nach dem Indexereignis erhielten 1018 Versicherte (1,2\% der Subgruppe 2) eine Rehabilitationsverordnung.

\section{Kardiale Resynchronisationstherapie (CRT)}

Von den Versicherten mit Stadium NYHA III und IV $(\mathrm{n}=37343)$ wurden innerhalb eines Jahres 3674 Personen (9,8\%) ein Schrittmacher oder Defibrillator implantiert.

\section{Einsetzen eines implantierbaren Cardioverter-}

Defibrillators nach überlebten Herzrhythmusstörungen

Es wurden 3573 Versicherte identifiziert, die im Studienzeitraum mindestens eine ICD-Diagnose hatten, die auf einen plötzlichen Herztod schließen lässt (ICD-10: I46,0, I46,1, R96.-). Laut Routinedaten wurde 536 (15\%) Patienten mit kodiertem plötzlichem Herztod ein Defibrillator implantiert. 


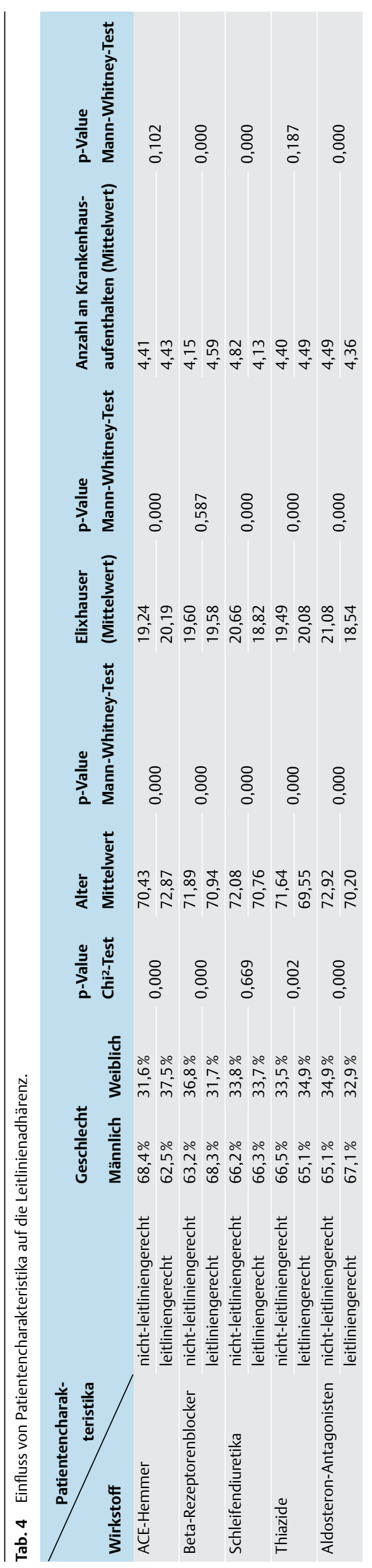

\section{Ärztliche Betreuung}

Über die LANR wurden die Versicherten in die Gruppen „ausschließlich vom Facharzt behandelt“ ( $n=266)$, „ausschließlich vom Hausarzt behandelt“ ( $n=25173$ ), „von beiden Arztgruppen behandelt“ ( $n=20654)$, und „weder vom Hausarzt noch von einem ausgewählten Facharzt behandelt“ ( $n=343)$ unterteilt.

\section{Einfluss von Patientencharakteristika auf die Leitlinienadhärenz}

Die Subgruppenanalysen zeigen signifikante Unterschiede für die jeweiligen Wirkstoffe beim Alter sowie teilweise bei Geschlecht, Komorbiditätsindex und bei der Anzahl an Krankenhausaufenthalten ( $\bullet$ Tab. 4). Die Analyse mittels logistischer Regression konnte jedoch aufgrund geringer Modellgüte keine weiteren Erkenntnisse liefern (Ergebnisse nicht dargestellt).

\section{Diskussion \\ $\nabla$}

In der vorliegenden Studie konnte gezeigt werden, dass sich eine Reihe von Leitlinienempfehlungen mithilfe von Routinedaten abbilden lassen. Voraussetzung ist, dass die Empfehlungen mittels EBM-Ziffern, ATC- und OPS-Codes oder ICD-10-Diagnosen definiert sowie zeitliche und mengenmäßige Angaben konkret formuliert waren sind. Am validesten gelang dies bei der Analyse der Pharmakotherapie, da für diese Informationen zu herzinsuffizienz-spezifischen Wirkstoffgruppen sowie Kontraindikationen vorliegen. In diesem Bereich konnte gezeigt werden, dass die Versorgungsquote bei den Beta-Rezeptorenblockern am höchsten lag, gefolgt von den Schleifendiuretika und den ACE-Hemmern.

Durch die mangelnde Abbildbarkeit von Lebensgewohnheiten konnten jedoch keine Aussagen über die bei der Herzinsuffizienz sehr wichtige Rolle der eigenverantwortlichen Etablierung eines gesunden Lebensstils durch den Patienten getroffen werden. Die Einbindung der DMP-Dokumentation wäre ein erster Schritt, um diese Limitation zu überwinden. Jedoch zeigen aktuelle Untersuchungen von Horenkamp-Sonntag et al., dass es Defizite in der Dokumentationsqualität gibt, was zu einer weiteren Herausforderung führt [16]. Auch die familiäre Disposition für bestimmte Erkrankungen, welche Risikofaktoren für die Herzinsuffizienz darstellen [17] und bei der Planung präventiver Maßnahmen Berücksichtigung finden sollten, kann nicht anhand von Routinedaten ermittelt werden.

Die Aussagekraft von Längsschnittanalysen zur Medikationsstrategie ist immer dann eingeschränkt, wenn Patienten im Zeitablauf stationär behandelt wurden, da bei stationären Krankenhaus- und Rehabilitationsaufenthalten verordnete Arzneimittel in GKV-Routinedaten aufgrund der pauschalierten Abrechnungssysteme nicht abgebildet werden. Ähnliches gilt für ausgewählte EBM- und OPS-Leistungen im ambulanten sowie stationären Bereich, da diese zum Teil pauschal abgerechnet werden. Einzelne Leistungen können daher nicht differenziert abgebildet werden was zu einer Unterschätzung der tatsächlich erbrachten Leistungen führen kann.

Der Anteil an Personen, die eine Rehabilitationsmaßnahme verschrieben bekommen haben, erscheint in dieser Studie recht gering. Ein Grund dafür ist, dass für die Erstattung von Rehabilitationen in Deutschland unterschiedliche Kostenträger zuständig sind. Bei jüngeren und erwerbsfähigen Versicherten ist in der Regel die Rentenversicherung zuständig, sodass für diesen Personenkreis keine Aussagen zum Rehabilitationsgeschehen mit- 
tels GKV-Routinedaten möglich sind [18]. Es kann somit nur ein geringer Anteil der Rehabilitationsmaßnahmen abgebildet werden.

Der Anteil derjenigen Patienten, die ein EKG bzw. eine Echokardiografie erhielten, fällt ebenfalls sehr gering aus. Möglicherweise werden diese Leistungen pauschal abgerechnet. $\mathrm{Zu}$ dieser Schlussfolgerung kommen auch Laux et al., die aus diesem Grund auf das Überprüfen der Qualitätsindikatoren EKG und Echokardiografie verzichtet haben [10]. Aus ihrer Sicht ist die Anzahl der Patienten mit EKG bisher nicht aus den Routinedaten ablesbar bzw. überprüfbar und es existiert auch keine einheitliche Dokumentationsvorschrift für dieses Leistungsangebot. Eine Echokardiografie wird laut Laux et al. über die sogenannte Leistungskomplexziffer abgerechnet [10].

Der Anteil implantierbarer Cardioverter-Defibrillatoren scheint ebenfalls sehr gering zu sein. Dies könnte darauf zurückzuführen sein, dass durch die Praxissoftware die Diagnose lediglich weitergeführt wurde und nicht als eine „Zustand-nach-Diagnose“ kodiert worden ist. Des Weiteren ist es möglich, dass Patienten mit plötzlichem Herztod schon bereits einen Defibrillator-Implantat hatten. Dieser Punkt könnte jedoch über die Kontrolle der EBM zur Wartung und Kontrolle der implantierten Aggregate adressiert werden.

Grippeschutzimpfungen sind möglicherweise - obwohl Herzinsuffizienz-Patienten zur Risikogruppe gehören - privat vom Versicherten oder im Rahmen der betrieblichen Gesundheitsförderung vom Arbeitgeber bezahlt worden und damit nicht in den Abrechnungsdaten der gesetzlichen Krankenkassen ersichtlich. Zusätzlich könnten KV-spezifische Sonderziffern statt EBM-Ziffern abgerechnet worden sein, da Präventionsleistungen je KV unterschiedlich kodiert sein können. Des Weiteren sind in Krankenhäusern durchgeführte Grippeschutzimpfungen nicht abbildbar, da diese unter die DRG-Pauschalen fallen und damit nicht separat abgerechnet werden.

Eine zusätzliche methodische Herausforderung besteht hinsichtlich der Pharmakotherapie und der Compliance des Patienten. So ist es möglich, dass, obwohl der Arzt leitliniengerecht behandelt und die Medikationsstrategie leitliniengerecht verordnet, der Patient aus bestimmten Gründen, z.B. durch Reaktanz gewisser Wirkstoffe, das Rezept nicht einlöst. Diese Verordnungen wären dann in den Routinedaten nicht ersichtlich, da nur in der Apotheke eingelöste Rezepte an die Krankenkassen übermittelt werden. Dieses Informationsdefizit könnte zu falschen Aussagen bezüglich des leitliniengerechten Verhaltens der Ärzte führen. Darüber hinaus könnte die Nichteinnahme der in der Apotheke erworbenen Medikamente durch den Patienten zu verzerrten Ergebnissen bezüglich der patientenrelevanten Endpunkte führen.

Unklar bleibt, ob der nicht nachgewiesene statistisch signifikante Zusammenhang zwischen Alter, Geschlecht, NYHA Status sowie Komorbiditäten und Leitlinienadhärenz in der Datengrundlage begründet oder tatsächlich kein Zusammenhang vorhanden ist. Hier besteht weiterer Forschungsbedarf.

Aufgrund der allgemeingeltenden Limitationen der GKV-Routinedaten [7,19], wie z. B. das nicht Vorhandensein von Symptomen und klinischen Befunden sowie der Ergebnisqualität konnten vereinzelte Leitlinienempfehlungen nicht abgebildet werden. Die Einteilung in „falsch“ verordnete Wirkstoffe wurde auf die mit ICD-10-Codes abbildbaren Kontraindikationen begrenzt. Es existieren jedoch weitere Kontraindikationen z.B. Intoleranzen, die meist klinischer Natur und dadurch mit GKV-Routinedaten nicht darstellbar sind.
Mit dem vorliegenden Studiendesign ist nicht ermittelbar, wie die Patienten vor dem 01.01.2008 behandelt worden sind. Problematisch wird dies z.B. bei der kardialen Resynchronisationstherapie, da nicht ersichtlich ist, ob der Versicherte zuvor schon eine Implantation eines Herzschrittmachers oder eines Defibrillators erhalten hat. Ein längerer Beobachtungszeitraum kann in den meisten Routinedatenstudien nicht analysiert werden, da die Krankenkassen in der Regel ihre Daten lediglich über einen Zeitraum von 4 Jahren vorhalten und zur Verfügung stellen dürfen. Es konnte gezeigt werden, dass GKV-Routinedaten spezifische Limitationen aufweisen, welche die Überprüfbarkeit von Leitlinienempfehlungen einschränken. Dennoch konnte eine Reihe von Empfehlungen identifiziert werden, welche sich valide anhand dieser Datengrundlage überprüfen lassen. Des Weiteren handelt es sich bei der NVL um eine Leitlinie und nicht um eine Richtlinie. So ist es legitim, dass Ärzte sich nicht an die Empfehlungen der Leitlinie halten, wenn dem Patienten eine andere Therapie aufgrund von individuellen Patientencharakteristika zuträglicher wäre und der Nutzen das Risiko übersteigt.

\section{Danksagungen}

Die vorliegende Studie wurde vom Zentralinstitut für die Kassenärztliche Versorgung (ZI) gefördert.

Interessenkonflikt: Das Wissenschaftliche Institut der TK für Nutzen und Effizienz im Gesundheitswesen (WINEG) hat zur Aufgabe, die Wertigkeit von Innovationen und neuen programmatischen Ansätzen innerhalb der GKV zu hinterfragen. Die Autoren Prof. Dr. Roland Linder, Dr. Susanne Engel und Dr. Frank Verheyen erklären, dass aufgrund ihrer Zugehörigkeit zur Techniker Krankenkasse ein potenzieller Interessenkonflikt im Sinne der Richtlinien des International Committee of Medical Journal Editors besteht. Die weiteren Autoren erklären, dass keine Interessenkonflikte bestehen.

\section{Literatur}

1 DEGAM. Leitlinie Nr. 9: Herzinsuffizienz. Langfassung. Düsseldorf: Omikron Publ; 2006

2 McMurray JJ. HEART FAILURE: Epidemiology, aetiology, and prognosis of heart failure. Heart 2000; 83: 596-602

3 NVL. Nationale Versorgungsleitlinie Chronische Herzinsuffizienz Langfassung. 2011

4 Statistisches Bundesamt. Gesundheit - Todesursachen in Deutschland 2011. Wiesbaden: 2012

5 Statistisches Bundesamt. Krankheitskostenrechnung. Im Internet: https://www-genesis.destatis.de/genesis/online/logon?language=de\& sequenz=tabellen\&selectionname $=23631^{*}$ Stand: 02.09 .2015

6 Hoffmann F. Review on use of German health insurance medication claims data for epidemiological research. Pharmacoepidem Drug Safe 2009; 18: 349-356

7 Zeidler J, Braun S. Sekundärdatenanalysen. In: Schöffski O, Graf von der Schulenburg J-M, (Hrsg.). Gesundheitsökonomische Evaluationen. Berlin, Heidelberg: Springer-Verlag Berlin Heidelberg; 2012: 243-274

8 Hasenbein $U$, Wallesch $C$. Was ist Leitlinienkonformität? Theoretische und methodische Überlegungen zu einem aktuellen Konzept der Versorgungsforschung und Qualitätssicherung. Gesundheitswesen 2007; 69: 427-437

9 Swart E, Willer C. Lässt sich die Umsetzung ärztlicher Leitlinien anhand von GKV-Routinedaten überprüfen? Gesundheitswesen 2012; 74

10 Laux G, Nothacker M, Weinbrenner S et al. Nutzung von Routinedaten zur Einschätzung der Versorgungsqualität: Eine kritische Beurteilung am Beispiel von Qualitätsindikatoren für die „Nationale Versorgungsleitlinie Chronische Herzinsuffizienz“. Zeitschrift für Evidenz, Fortbildung und Qualität im Gesundheitswesen 2011; 105: 21-26 
11 Flesch $M$, Komajda $M$, Lapuerta $P$ et al. Leitliniengerechte Herzinsuffizienzbehandlung in Deutschland. Dtsch med Wochenschr 2005; 130 : 2191-2197

12 Deutsche Angestellten Krankenkasse (DAK). Unterversorgung bei Herzinsuffizienz. Leitliniengerechte Therapie - für jeden dritten Patienten mit Herzinsuffizienz bisher nur Theorie/DAK-Daten zeigen bessere Versorgungsqualität von DMP-Teilnehmern 2011

13 Schubert I, Köster I, Küpper-Nybelen J et al. Versorgungsforschung mit GKV-Routinedaten. Bundesgesundheitsbl 2008; 51: 1095-1105

14 Hoppe UC, Böhm M, Dietz R et al. Leitlinien zur Therapie der chronischen Herzinsuffizienz. ZS Kardiologie 2005; 94: 488-509

15 Deutsches Ärzteblatt. (Hrsg.). Techniker Krankenkasse überrundet Barmer-GEK. Im Internet: http://www.aerzteblatt.de/nachrichten/57202/ Techniker-Krankenkasse-ueberrundet-Barmer-GEK 2014

16 Horenkamp-Sonntag D, Linder R, Ahrens $S$ et al. Externe Validität von DMP-Doku-Bögen im Abgleich mit GKV Routinedaten: Wie valide werden Arzneimittel-Therapien und stationäre Notfalleinweisungen von DMP-Ärzten dokumentiert? Universität Konstanz 2012
17 Kilter $H$. Diagnose und Differenzialdiagnose der Herzinsuffizienz. In: Bäumer AT, Böhm M, (Hrsg.). Herzinsuffizienz. 55 Tabellen. Stuttgart [u.a.]: Thieme; 2000: 48-53

18 Zeidler J, Mittendorf T, Vahldiek G et al. Kostenvergleichsanalyse der ambulanten und stationären kardiologischen Rehabilitation. Herz 2008; 33: 440-447

19 Neubauer S, Zeidler J, Lange A et al. Grundlagen und Methoden von GKV-Routinedatenstudien. Diskussionspapier. Leibniz Universität Hannover - Center for Health Economics Research Hannover (CHERH). 2014; No. 534. Im Internet: http://diskussionspapiere.wiwi.uni-han nover.de/pdf_bib/dp-534.pdf 